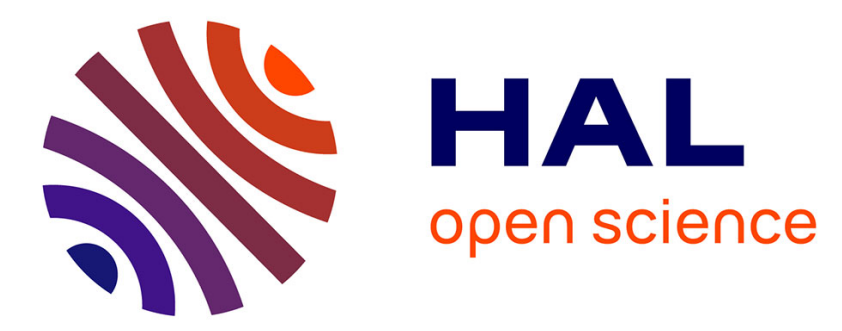

\title{
Transition to chaos for two-frequency systems
}

\author{
R.S. Mackay, C. Tresser
}

\section{To cite this version:}

R.S. Mackay, C. Tresser. Transition to chaos for two-frequency systems. Journal de Physique Lettres, 1984, 45 (15), pp.741-746. 10.1051/jphyslet:019840045015074100 . jpa-00232406

\section{HAL Id: jpa-00232406 https://hal.science/jpa-00232406}

Submitted on 1 Jan 1984

HAL is a multi-disciplinary open access archive for the deposit and dissemination of scientific research documents, whether they are published or not. The documents may come from teaching and research institutions in France or abroad, or from public or private research centers.
L'archive ouverte pluridisciplinaire HAL, est destinée au dépôt et à la diffusion de documents scientifiques de niveau recherche, publiés ou non, émanant des établissements d'enseignement et de recherche français ou étrangers, des laboratoires publics ou privés. 


\title{
LE JOURNAL DE PHYSIQUE-LETTRES
}

J. Physique Lett. 45 (1984) L-741 - L-746

1er AOÛT 1984, PAGE L-741

Classification

Physics Abstracts

05.40

\section{Transition to chaos for two-frequency systems}

\author{
R. S. MacKay $(*)$ and C. Tresser $(* *)$ \\ Institut des Hautes Etudes Scientifiques, 35, route de Chartres, 91440 Bures-sur-Yvette, France
}

(Reçu le 10 mai 1984, accepté le 7 juin 1984)

\begin{abstract}
Résumé. - De nombreux flots bi-périodiques peuvent être modélisés par des applications du cercle dans lui-même. Nous décomposons en quatre composantes, la frontière du chaos topologique dans l'espace des applications $\mathrm{C}^{1}$ de degré 1 du cercle, et nous décrivons les routes typiques vers le chaos topologique.
\end{abstract}

\begin{abstract}
Many biperiodic flows can be modelled by maps of a circle to itself. We decompose the boundary of topological chaos in the space of $\mathrm{C}^{1}$ circle maps of degree 1 into four subsets, and describe the typical routes to topological chaos.
\end{abstract}

In many experiments on chaotic dynamics, both physical (e.g. [1] and references therein) and computational [2-4], one sees a sequence of behaviour on varying a parameter, which can be described in the phase space as follows :

\begin{tabular}{|c|c|c|}
\hline \multicolumn{3}{|l|}{ flows : ATTRACTING 1} \\
\hline EQUILIBRIUM - & PERIODIC ORBI' & $\rightarrow$ TWO-TORUS $\rightarrow$ \\
\hline (return)maps & ATTRACTING & 2 ATTRACTING 3 «CHAOS » \\
\hline & FIXED POINT & CIRCLE \\
\hline
\end{tabular}

Our interest is in transition 3 in cases for which the dynamics on the attracting circle can be described by an orientation preserving circle homeomorphism (as when transitions 1 and 2 are " Hopf » bifurcations). Although this has been greatly studied [2-7], one is still far from a complete classification of the possible routes.

Maps of a circle to itself have been proposed [8], and widely used [3,7,9-13], to model many systems exhibiting this transition.

(*) Permanent address : Mathematics Institute, University of Warwick, Coventry, CV4 7AL, England.

(**) Permanent address : Physique de la Matière Condensée, LA 190, Parc Valrose, 06034 Nice Cedex, France. 
Remark 1. - All (return) maps having a contracting ring not containing two disjoint ones, are expected to reduce in some sense to circle maps. Here we say that $A \subset M$ is a contracting ring for a map $f: \mathbf{M} \rightarrow \mathbf{M}$, if $\mathbf{A}$ is topologically a circle cross a codimension one closed disk, and is mapped strictly inside itself. This covers a large class of systems exhibiting transition 3 , though it does exclude some cases of interest $[14,15]$.

In this Letter, we classify all routes to chaos for a class of circle maps. More precisely, we decompose the boundary of topological chaos in the space $C^{1}\left(\mathbb{T}^{1}\right)$ of degree one, $\mathbf{C}^{1}$ (i.e. continuously differentiable) circle maps into four well-characterized subsets, and describe the typical ways it can be crossed. First we need to define topological chaos and the concepts of lifts, degree, rotation number and frequency locking for circle maps.

We say a map $f$ has topological chaos if, given any accuracy of observation, there exist a strictly increasing sequence of integers $n_{i}$ and a real number $h>0$ such that the number of distinguishable orbit segments of length $n_{i}$ grows asymptotically like $\exp \left(n_{i} h\right)$. The largest such $h$ is called the topological entropy $h(f)$ [16]. Although topological chaos does not necessarily imply complicated asymptotic behaviour of most orbits, it is a prerequisite.

Given a continuous map $f$ of the circle $\mathbb{T}^{1}=\mathbb{R} / \mathbb{Z}$ to itself, it is useful to consider its lifts to continuous maps $F$ of the line $\mathbb{R}$ to itself, i.e. those maps such that $f \circ \pi=\pi \circ F$, where $\pi(x)=$ $x(\bmod 1)$ is the canonical projection from $\mathbb{R}$ to $\mathbb{T}^{1}$. Different lifts differ only by an integer constant. $F(x+1)-F(x)$ is an integer constant, known as the degree of $f$. We consider only degree one circle maps, since this is the case that arises by deforming orientation preserving homeomorphisms.

Given a lift $F$ of a circle map, we say a point $x$ has rotation number $\rho(x)=\lim _{n \rightarrow \infty} \frac{1}{n}\left(F^{n}(x)-x\right)$ if the limit exists. Given $F$, the set of all rotation numbers is always a single number or a closed interval $[6,17,18]$, which we call its rotation interval $\rho(F)$, and is translated by an integer if one chooses a different lift of the same circle map. If $\rho(F)$ is a single rational $\omega$, we say $F$ is frequency locked with ratio $\omega$.

In the course of this Letter we will define various sets of circle maps. Their relative disposition is illustrated in figure 1, which we recommend the reader keep continually in view.

Remark 2. - Because there are two main ingredients in their dynamics, namely the nonlinearity and the rotation number, we represent spaces of circle maps by two dimensional pictures, As a guide, think of two parameter families like

$$
F_{a, b}(x)=x+a-\frac{b}{2 \pi} \sin 2 \pi x .
$$

Firstly, we denote by $\mathrm{L}_{\omega}$ the set of frequency locked maps with ratio $\omega$, and define the $\omega$-Arnold tongue $\mathrm{A}_{\omega}$ to be the set of all maps $F$ such that $\omega \in \rho(F)$. We extend the definitions of $\mathrm{L}_{\omega}$ and $\mathrm{A}_{\omega}$ to $\omega$ irrational, though then the names are somewhat inappropriate. For any $\omega \in \mathbb{R}, L_{\omega}$ is strictly contained in $\mathrm{A}_{\omega}$, but both notions coincide for monotone maps, as illustrated in figure 1 . The main properties of these sets are described in [19] $\left({ }^{1}\right)$.

Remark 3. - For $\omega$ irrational, the possibility of $F \in \mathrm{L}_{\omega}$ but non-monotone, which is somewhat pathological, can be eliminated by considering only analytic maps or by restricting $\mathrm{C}^{1}\left(\mathbb{T}^{1}\right)$ slightly to a certain class $\mathrm{A}^{1}\left(\mathrm{~T}^{1}\right)[18,19]$.

Next, we define $Z_{p / q}$ to be the subset of $A_{p / q}$ consisting of those maps for which all periodic orbits of rotation number $p / q$ have period of the form $q .2^{n}$, for some $n$ (all fractions are supposed to be written in lowest terms).

Now we are ready to describe the boundary of topological chaos in $\mathrm{C}^{1}\left(\mathbb{T}^{1}\right)$.

( ${ }^{1}$ ) See also Boyland [20] for an independent treatment of these notions in a subclass of $\mathrm{C}^{1}\left(\mathbb{T}^{1}\right)$. 


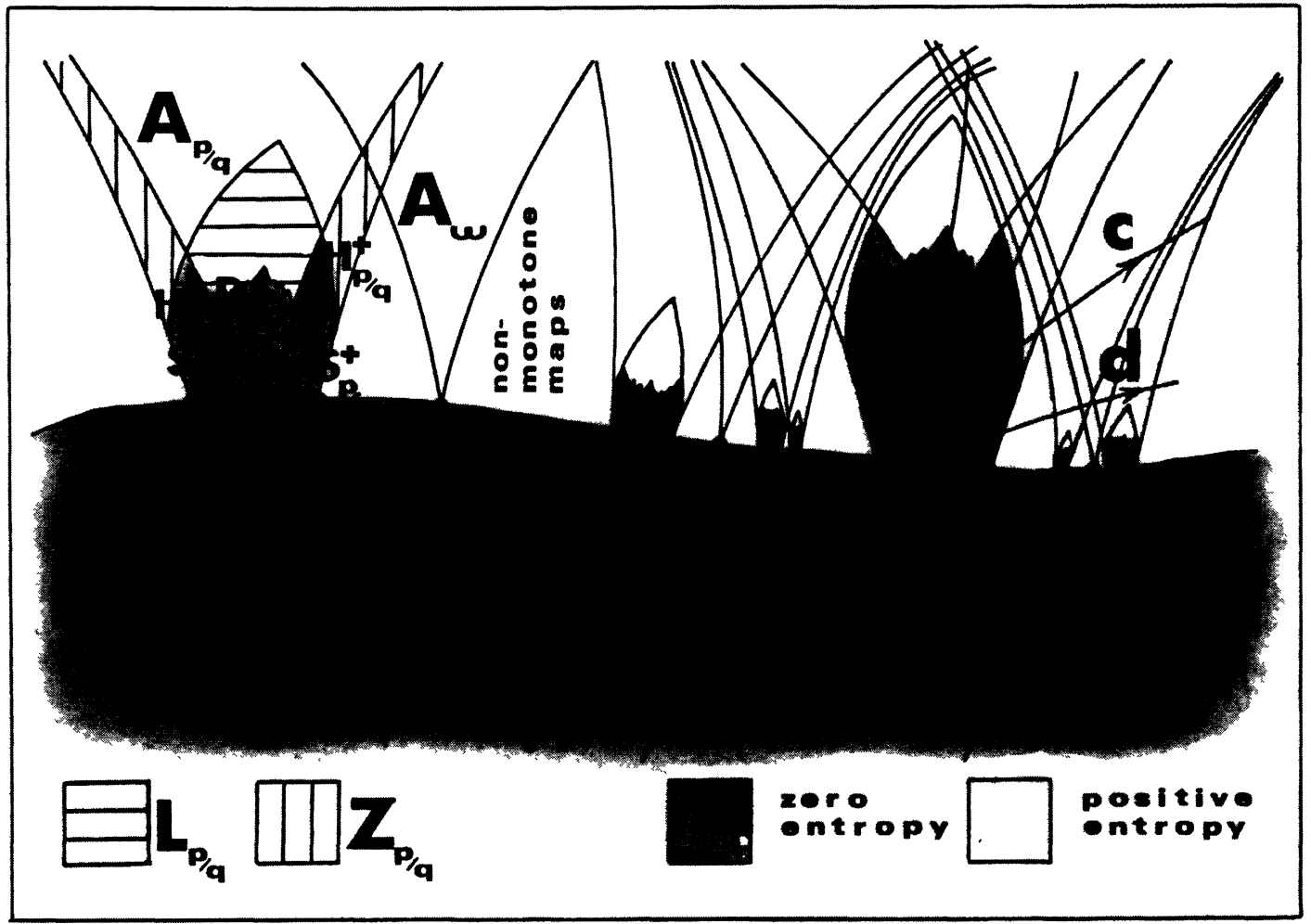

Fig. 1. - A two-dimensional representation of the space $A^{1}\left(\mathbb{T}^{1}\right)$ (see Remark 3), illustrating various relevant subsets and their boundaries. We show four sample paths whose characteristics are illustrated in figure 2. The figure is also intended to indicate : the opening of irrational tongues at the boundary of non-invertibility in class $A^{1}\left(\mathbb{T}^{1}\right)$, the fact that all boundary points of $L_{p / q}$ are accumulation points of other tongues, and the complex structure of part $D_{p / q}$ of the boundary of chaos, which will be described in [26] in terms of kneading theory for families which look like $F_{a, b}$.

Remark 4. - It is good to impose as weak smoothness conditions as possible, since the induced circle map for the contracting ring need not be smooth. Some smoothness, however, is necessary for the transition problem to make sense, since any continuous circle map can be approximated by continuous circle maps with positive topological entropy [33].

Theorem [19]. - The boundary of topological chaos in $C^{1}\left(\mathbb{T}^{1}\right)$ is the union of the following four subsets :

$$
\begin{aligned}
& S=\bigcup_{p / q \in \mathbf{Q}} S_{p / q} \text {, with } S_{p / q} \\
& =\{\text { non-monotone maps in the common part of the boundaries } \\
& \text { of } \left.A_{p / q} \text { and } L_{p / q}\right\} \text {. } \\
& H=\bigcup_{p / q \in Q} H_{p / q} \text { with } H_{p / q}=\left\{\begin{array}{l}
\text { maps in the boundary between } L_{p / q} \text { and the rest of } A_{p / q} \\
\text { belonging also to } Z_{p / q} \text { \}. }
\end{array}\right.
\end{aligned}
$$

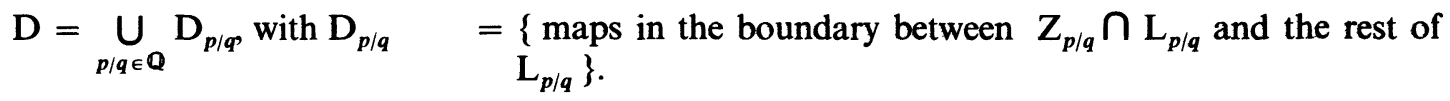

$$
\begin{aligned}
& I=\left(\bigcup_{\omega \in \mathbf{R} / \mathbf{Q}} I_{\omega}\right) U\left(\bigcup_{p / q \in \mathbf{Q}} I_{p / q}\right) \\
& \text { with } \mathrm{I}_{\omega}=\left\{\text { non-diffeomorphisms in } \mathrm{L}_{\omega}\right\} \\
& \text { and } I_{p / q}=\left\{\text { monotone non-diffeomorphisms on the boundary of } A_{p / q}\right\} \text {. }
\end{aligned}
$$


Remark 5. - It is convenient furthermore to decompose $\mathrm{S}_{p / q}$ into $\mathrm{S}_{p / q}^{+} \cup \mathrm{S}_{p / q}^{-}$, the sign indicating whether larger or smaller rotation numbers may be found on crossing that part of the boundary ; similarly for $\mathrm{H}_{p / q}$ and $\mathrm{I}_{p / q}$. The relative disposition of all these sets is shown in figure 1 .

To each of the four parts of the boundary corresponds a characteristic route to chaos for " neat " crossings. Different routes yield different evolution of the rotation interval; all give positive topological entropy. For generic one parameter families, one sees only the following routes, and $\rho(F)$ and $h(f)$ evolve as indicated. We denote by $\varepsilon$ the difference in parameter from the critical case, and the $C_{j}$ represent constants, $C_{1}, C_{2}>0, C_{3}<C_{4}$.

Route $\mathrm{S}_{p / q^{*}}^{ \pm}$- The transition is marked by a Saddle-node which destroys the last orbits with rotation number $p / q$, and one obtains a non-trivial rotation interval $\rho(F)>p / q\left(\mathrm{~S}_{p_{1}}^{+}\right)$or $<p / q\left(\mathrm{~S}_{p / q}^{-}\right)$:

$$
\rho\left(F_{\varepsilon}\right) \sim \frac{p}{q} \pm C_{1} \varepsilon^{1 / 2}+\left[C_{3} \varepsilon, C_{4} \varepsilon\right], \text { respectively } ; h\left(f_{\varepsilon}\right) \gtrsim C_{2} \varepsilon
$$

Route $\mathrm{H}_{p / q^{\circ}}^{ \pm}$- The transition $;$marked by creation of a Heteroclinic loop in the sense of [22], giving a non-trivial rotation inte ' with $p / q$ as left or right endpoint, respectively. This involves either a heteroclinic tangency in the sense of [23] (as for the family $F_{a, b}$ [20]), or a saddle-node. In the first case, the other endpoint of $\rho\left(F_{\varepsilon}\right)$ evolves like :

$$
\frac{p}{q} \pm \frac{C_{1}}{\log 1 / \varepsilon}, \text { respectively; and } h\left(f_{\varepsilon}\right) \gtrsim C_{2}\left(\log \frac{1}{\varepsilon}\right)^{-1 / 2}
$$

and in the second case, like :

$$
p / q \pm C_{1} \varepsilon^{1 / 2}, \text { respectively; and } h\left(f_{\varepsilon}\right) \gtrsim C_{2} \varepsilon^{1 / 4} .
$$

Route $\mathrm{D}_{p / q}$ : Accumulation of period Doubling bifurcations with periods $q .2^{n}: \rho(F)$ remains equal to $\{p / q\}$, and $h\left(f_{\varepsilon}\right) \propto \varepsilon^{\frac{\log 2}{\log \delta}}, \delta \simeq 4.6692$ (provided the crossing occurs in the stable manifold of the known quadratic fixed point $[24,25]$ of the doubling operator).

Remark 6. - Route I does not appear in our list because it is not stable to perturbation in one parameter families. It is stable, however, in two parameter families. Also $D_{p / q}$ has special points, where different scaling is to be expected, which cannot be avoided in non-trivial two parameter families.

Further details and justifications of all these results will be given in references $[19,26]$.

Routes $\mathrm{S}$ and $\mathrm{D}$ have already been recognized in experiments [1]. Route $\mathrm{H}$ is new, but need not have immediate consequences for experiments since in general, crossing $\mathrm{H}_{p / q}$ does not remove the stable periodic orbits present in $\mathrm{L}_{p / q} \cap \mathrm{Z}_{p / q}$. However, the crossing creates other attracting sets, some of which are typically chaotic. These may make themselves evident on jogging the system or by hysteresis $[19,27]$.

Both $\rho(F)$ and $h(f)$ have implications for experimentally measurable quantities e.g. the rotation interval of a point $x[28]$ :

$$
\rho(x, F)=\left[\rho_{-}(x, F), \rho_{+}(x, F)\right] \quad \text { with } \quad \rho_{ \pm}(x, F)=\lim _{n \rightarrow \infty} \sup _{\inf } \frac{F^{n}(x)-x}{n},
$$

and the Lyapunov exponent $\lambda(f)$ (when well defined : see e.g. [29] and references therein). One has

and

$$
\rho_{-}(F) \leqslant \rho_{-}(x, F) ; \rho_{+}(x, F) \leqslant \rho_{+}(F)
$$

$$
\lambda(f) \leqslant h(f) .
$$


Although the bounds are not necessarily attained, we expect $\rho(x . F)$ and $\lambda(f)$ to show similar scaling behaviours to $\rho(F)$ and $h(f)$, respectively.

Figure 2 shows the behaviour of $\rho(F)$ and $h(f)$ for four typical paths indicated on figure 1 . The results of many experiments in which biperiodic behaviour leads to chaos can be interpreted

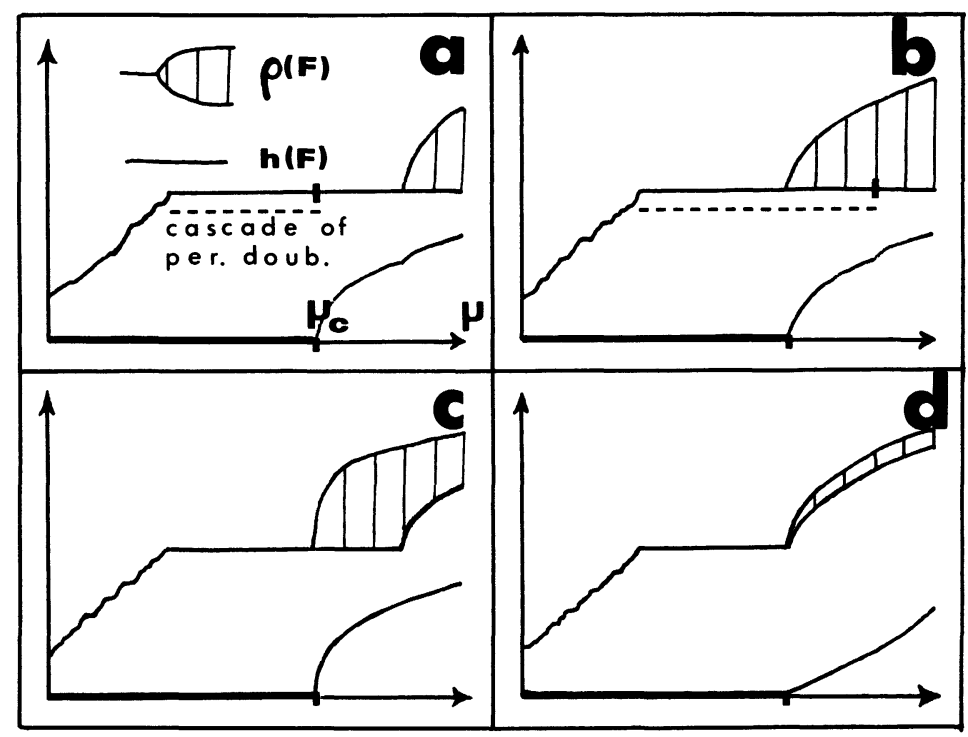

Fig. 2. - Behaviour of $\rho\left(F_{\mu}\right)$ and $h\left(f_{\mu}\right)$ for one parameter families following the paths indicated in figure 1. In each case, $\mu_{\mathrm{c}}$ denotes the parameter value where the family crosses the boundary of topological chaos.

by paths like these, or more complicated ones. Thus we propose figure 1 as a useful tool in understanding this transition.

\section{Acknowledgments.}

We would like to thank IHES for their warm hospitality.

\section{References}

[1] Gollub, J. P. and LibChaber, A., in Chaotic behaviour of deterministic systems, Les Houches 1981,

G. Iooss, R. H. G. Helleman and R. Stora, eds. (North Holland) 1983.

[2] Curry, J. H. and Yorke, J. A., in Springer Lecture Notes in Math. no 668 (1978).

[3] Coullet, P., Tresser, C. and Arnéodo, A., Phys. Lett. A 77 (1980) 327.

[4] Aronson, D. G., Chory, M. A., Hall, G. R. and R. P. McGehiee, R. P., Commun. Math. Phys. 83 (1982) 303.

[5] LEVI, M., Met. A.M.S. no 214 (1981).

[6] Newhouse, S., Palis, J. and Takens, F., Publ. Math. IHES 57 (1983) 5.

[7] Rand, D., Ostlund, S., Sethna, J. and Siggia, E. D., Physica D 8 (1983) 303.

[8] GuCKenheimer, J., Physica D 1 (1980) 227.

[9] Shenker, S. J., Physica D 5 (1982) 405.

[10] Feigendaum, M. J., Kadanoff, L. P. and Shenker, S. J., Physica D 5 (1982) 370. 
[11] Glass, L. and Perez, R., Phys. Rev. Lett. 48 (1982) 1772.

[12] Perez, R. and Glass, L., Phys. Lett. A 90 (1982) 441.

[13] Schell, M., Fraser, S. and Kapral, R., Phys. Rev. A 28 (1983) 373.

[14] Chenciner, A., in Chaotic behaviour of deterministic systems, Les Houches 1981, G. Iooss, R. H. G. Helleman and R. Stora, eds. (North Holland) 1983.

[15] Guckenheimer, J. and Holmes, P., Nonlinear Oscillations, Dynamical Systems, and Bifurcations of Vector Fields (Springer) 1983.

[16] BowEN, R., Regional conferences series in Math. $\mathrm{n}^{\circ} 35$ (1978).

[17] Ito, R., Math. Proc. Camb. Phil. Soc. 89 (1981) 107.

[18] Chenciner, A., Gambaudo, J. M. and Tresser, C., Une remarque sur la structure des endomorphismes de degré 1 du cercle. Preprint IHES/P/84/16.

[19] MacKay, R. S. and Tresser, C., Transition to topological chaos for circle maps. In preparation.

[20] Boyland, P. L., Thesis, Boston (1983).

[21] YANO, K., Inventiones Math. 59 (1980) 215.

[22] Block, L., Proc. A.M.S. 72 (1978) 576.

[23] VAN Strien, S. J., in Springer Lecture Notes in Math. $\mathrm{n}^{\circ} 898$ (1981).

[24] Campanino, M., Epstein, H. and Ruelle, D., Topology 21 (1982) 125.

[25] LANFord III, O., Bull. Amer. Math. Soc. 6 (1983) 427.

[26] MACKAY, R. S. and TRESSER, C., Kneading theory for some maps with two critical points. In preparation.

[27] Arnéodo, A., Coullet, P., Tresser, C., Libchaber, A., Maurer, J. and D'Humières, D., Physica D 6 (1983) 305.

[28] Bamon, R., Malta, I. P., Pacifico, M. J. and Takens, F., Rotation intervals of endomorphisms of the circle. Preprint Groningen (1983).

[29] Ruelle, D., Publ. Math. IHES 50 (1979) 27. 